\title{
Enhanced dielectric and electrocaloric properties in lead-free rod-like BCZT ceramics
}

\author{
Zouhair HANANI ${ }^{a, b}$, Soukaina MERSELMIZ ${ }^{a}$, Abdelaadim DANINE ${ }^{c}$, Nicolas \\ STEIN $^{c}$, Daoud MEZZANE ${ }^{a}$, M'barek AMJOUD $^{a,{ }^{*}}$, Mohammed LAHCINI ${ }^{a, d}$, \\ Yaovi GAGOU ${ }^{e}$, Matjaz SPREITZER ${ }^{f}$, Damjan VENGUST $^{f}$, Zdravko KUTNJAK ${ }^{f}$, \\ Mimoun El MARSSI ${ }^{e}$, Igor A. LUK'YANCHUK ${ }^{e, g}$, Mohamed GOUNÉ ${ }^{b}$ \\ ${ }^{a}$ IMED-Lab, Cadi Ayyad University, Marrakesh 40000, Morocco \\ ${ }^{b} I C M C B$, University of Bordeaux, Pessac 33600, France \\ ${ }^{c} I J L$, University of Lorraine, Nancy 54000, France \\ ${ }^{d}$ UM6P, Ben Guerir 43150, Morocco \\ ${ }^{e}$ LPMC, University of Picardy Jules Verne, Amiens 80039, France \\ ${ }^{f}$ Jozef Stefan Institute, Ljubljana 1000, Slovenia \\ ${ }^{g}$ Physics Faculty, Southern Federal University, Rostov-on-Don 344090, Russia
}

Received: October 29, 2019; Revised: December 24, 2019; Accepted: January 12, 2020

(C) The Author(s) 2020.

\begin{abstract}
Ba}_{0.85} \mathrm{Ca}_{0.15} \mathrm{Zr}_{0.10} \mathrm{Ti}_{0.90} \mathrm{O}_{3}$ (BCZT) lead-free ceramics demonstrated excellent dielectric, ferroelectric, and piezoelectric properties at the morphotropic phase boundary (MPB). So far, to study the effect of morphological changes on dielectric and ferroelectric properties in lead-free BCZT ceramics, researchers have mostly focused on the influence of spherical grain shape change. In this study, BCZT ceramics with rod-like grains and aspect ratio of about 10 were synthesized by surfactant-assisted solvothermal route. X-ray diffraction (XRD) and selected area electron diffraction (SAED) performed at room temperature confirm the crystallization of pure perovskite with tetragonal symmetry. Scanning electron microscopy (SEM) image showed that BCZT ceramics have kept the 1D rod-like grains with an average aspect ratio of about 4. Rod-like BCZT ceramics exhibit enhanced dielectric ferroelectric $\left(\varepsilon_{\mathrm{r}}=11,906, \tan \delta=0.014, P_{\mathrm{r}}=6.01 \mu \mathrm{C} / \mathrm{cm}^{2}\right.$, and $\left.E_{\mathrm{c}}=2.46 \mathrm{kV} / \mathrm{cm}\right)$, and electrocaloric properties $(\Delta T=0.492 \mathrm{~K}$ and $\zeta=0.289(\mathrm{~K} \cdot \mathrm{mm}) / \mathrm{kV}$ at $17 \mathrm{kV} / \mathrm{cm})$ with respect to spherical BCZT ceramics. Therefore, rod-like BCZT lead-free ceramics have good potential to be used in solid-state refrigeration technology.
\end{abstract}

Keywords: lead-free $\mathrm{Ba}_{0.85} \mathrm{Ca}_{0.15} \mathrm{Zr}_{0.10} \mathrm{Ti}_{0.90} \mathrm{O}_{3}$ (BCZT); rod-like $\mathrm{Ba}_{0.85} \mathrm{Ca}_{0.15} \mathrm{Zr}_{0.10} \mathrm{Ti}_{0.90} \mathrm{O}_{3}$ (BCZT); dielectric; ferroelectric; electrocaloric effect

\section{Introduction}

Due to the constant expansion of industry, increasing

* Corresponding author.

E-mail:m.amjoud@uca.ac.ma living standard, and climate change, the refrigeration market has become an increasingly significant factor in energy consumption [1-3]. The extensive use of refrigeration is one of the major factors of excessive energy consumption leading to the exhaustion of non-renewable energy resources, and thus deepening 
the greenhouse effect. Hence, developing sustainable technological solutions based on promising new cooling technologies is getting increasingly important in recent years. Several efficient techniques are under development such as solar [4], thermoelectric [5], magnetocaloric [6], and electrocaloric cooling [7]. The electrocaloric effect (ECE) has recently shown great potential, as the high electric fields required for the refrigeration cycle are easier and less expensive to produce than the other fields required in competitive refrigeration techniques [8,9]. Giant electrocaloric effect was found in lead-based ferroelectric ceramics like $\mathrm{Pb}(\mathrm{Zr}, \mathrm{Ti}) \mathrm{O}_{3}(\mathrm{PZT})$ and $\mathrm{Pb}(\mathrm{Zr}, \mathrm{Sn}, \mathrm{Ti}) \mathrm{O}_{3}$, as well as in relaxors such as $\mathrm{Pb}(\mathrm{Sc}, \mathrm{Nb}) \mathrm{O}_{3}$ and $\mathrm{Pb}\left(\mathrm{Mg}_{1 / 3} \mathrm{Nb}_{2 / 3}\right) \mathrm{O}_{3}-$ $\mathrm{PbTiO}_{3}$ (PMN-PT) [10-13]. However, the possible toxicity of lead-based materials limits their application in future eco-friendly cooling systems [3]. Correspondingly, new lead-free materials were developed recently to mimic the properties of lead-based materials [14-16].

Barium titanate $\left(\mathrm{BaTiO}_{3}\right)$ is usually regarded as one of potentially promising lead-free ceramics, which was the first material used to fabricate piezoelectric ceramics. It is widely employed in modern technologies such as mobile electronic devices and hybrid electrical vehicles $[17,18]$. Furthermore, $\mathrm{BaTiO}_{3}$ is a bioceramic material without any toxic or volatile elements, and its properties can be easily tailored by site engineering $[14,19]$. However, $\mathrm{BaTiO}_{3}$ exhibits low dielectric properties compared to lead-based materials and its dielectric constant below the Curie temperature $\left(T_{\mathrm{c}}=\right.$ $393 \mathrm{~K}$ ) rapidly drops due to the tetragonal/cubic phase transition [20,21]. To overcome the drawback, the $\mathrm{Ba}_{1-x} \mathrm{Ca}_{x} \mathrm{Zr}_{y} \mathrm{Ti}_{1-y} \mathrm{O}_{3}$ system is engineered by introducing $\mathrm{Ca}^{2+}$ and $\mathrm{Zr}^{4+}$ into the crystal lattice of $\mathrm{BaTiO}_{3}$, to replace $\mathrm{Ba}^{2+}$ and $\mathrm{Ti}^{4+}$, respectively, with improved dielectric properties due to enhanced relaxor behavior of $\mathrm{Ba}_{1-x} \mathrm{Ca}_{x} \mathrm{Zr}_{y} \mathrm{Ti}_{1-y} \mathrm{O}_{3}$ ceramics [22-26]. Moreover, as reported by Liu and Ren [27], $\mathrm{Ba}_{0.85} \mathrm{Ca}_{0.15} \mathrm{Zr}_{0.10} \mathrm{Ti}_{0.90} \mathrm{O}_{3}$ (BCZT) phase exhibits interesting dielectric, ferroelectric, and piezoelectric properties at the morphotropic phase boundary (MPB), which is comparable to those of PZT ceramics [15].

It is well known that the electrical properties of any material are very sensitive to its morphology. Almost all published articles on BCZT ceramics studied the dielectric and ferroelectric properties of sphericalgrain-based powder [28-30]. Hence, to correlate the electrical and morphological properties, researchers are focusing especially on spherical grain size effect. We reported previously, the effect of grain size and grain size distribution in BCZT ceramics using surfactantassisted solvothermal processing [31]. We found that the nature of surfactant directly influences the grain size and grain size distribution of BCZT ceramics. The use of sodium dodecyl sulfate ( $\mathrm{SDS}, \mathrm{NaC}_{12} \mathrm{H}_{25} \mathrm{SO}_{4}$ ) as surfactant results in highly dense ceramics with homogeneous grain distribution. However, cetyltrimethylammonium bromide ( $\left.\mathrm{CTAB}, \mathrm{C}_{19} \mathrm{H}_{42} \mathrm{BrN}\right)$ surfactant produces $\mathrm{BCZT}$ ceramics with bimodal grain size distribution including coarse grains and small spherical ones.

The surfactant-assisted solvothermal synthesis provides a design of nanomaterials with specifically tailored architectures via the synthesis of nanoscale building blocks with an appropriate size and shape, and controlled orientation of the final products. Moreover, the cationic surfactant $\mathrm{CTAB}$, a templating micelle molecule, is used to adjust size and morphology of nanomaterials [32-35]. At the best of our knowledge, there have been no other reports on the electrical studies in rod-like BCZT ceramics. In this contribution, we report the first BCZT ceramics with rod-like grains and evaluate its dielectric, ferroelectric, and electrocaloric properties.

\section{Experimental}

\section{1 Rod-like BCZT powder synthesis}

BCZT pure nanocrystalline powder was obtained by CTAB-assisted solvothermal synthesis as reported previously by Ref. [31]. First, an appropriate amount of barium acetate was dissolved in glacial acetic acid. Calcium nitrate tetrahydrate and zirconyl chloride octahydrate were dissolved each alone in 2-methoxyethanol. Second, the three solutions were mixed in a two-neck round bottom flask. In the third stage, titanium ${ }^{(\mathrm{IV})}$ isopropoxide $\left(\mathrm{Ti}\left(\mathrm{O}^{i} \mathrm{Pr}\right)_{4}\right)$ was added synchronously dropwise to the reaction medium using an isobaric dropping funnel.

Unlike the previously Ref. [31], to control BCZT microstructure, a different concentration $(30 \mathrm{mM})$ of CTAB surfactant was introduced. The obtained solution was transferred to a $30 \mathrm{~mL}$ teflon-lined stainless-steel autoclave at $180{ }^{\circ} \mathrm{C}$ in an oven for $12 \mathrm{~h}$. After the reaction was completed, the sealed autoclave was cooled in the air. The resulting product was washed sequentially with ethanol for several times. Then, the final product was dried at $100{ }^{\circ} \mathrm{C}$ for $12 \mathrm{~h}$, calcined at 
$1000{ }^{\circ} \mathrm{C}$ for $4 \mathrm{~h}$. Finally, BCZT powder was uniaxially pressed into pellets of diameter of about $6 \mathrm{~mm}$ and thickness of about $1 \mathrm{~mm}$, and sintered at $1250{ }^{\circ} \mathrm{C} /$ $10 \mathrm{~h}$ for electrical measurements. For $P-E$ loop measurements, the thickness of the sample was reduced to $0.4 \mathrm{~mm}$ to allow high electric field application, while the electrode area was kept to $28.26 \mathrm{~mm}^{2}$.

\section{2 Characterization rod-like BCZT}

The morphology of BCZT powder was investigated by a field-emission scanning electron microscope (FESEM, JEOL JSM-7600F). Crystalline structure of BCZT powder was performed by X-ray diffraction (XRD, Panalytical X-Pert Pro). The measurement has been done at room temperature employing a step angle of $0.017^{\circ}$ in the $2 \theta$ range from $10^{\circ}$ to $80^{\circ}$ using a $\mathrm{Cu} \mathrm{K} \alpha$ radiation $(\lambda \approx 1.540598 \AA$ ). The selected area of electronic diffraction (SAED) pattern was recorded by using a transmission electron microscope (TEM, Philips CM200). To determine the optimal calcination temperature of raw BCZT material in order to produce highly pure BCZT powder, the combined thermogravimetric and differential thermal analyses (TG-DTA, Sytram LABSYS evo) were performed at heating rate of $10{ }^{\circ} \mathrm{C} / \mathrm{min}$ from room temperature to $1100{ }^{\circ} \mathrm{C}$ in air. The resulting microstructure of BCZT sintered ceramics was analyzed by using a scanning electron microscope (SEM, Tescan VEGA3). The density of the sintered ceramics was measured by Archimedes method using deionized water as medium. A precision LCR meter (HP 4284A, $20 \mathrm{~Hz}$ to $1 \mathrm{MHz}$ ) was used to measure the dielectric properties of BCZT ceramic pellet that was electroded with silver paste in the frequency range of $100 \mathrm{~Hz}-1 \mathrm{MHz}$. The ferroelectric hysteresis loops were determined by using a ferroelectric test system (AiXACCT, TF Analyzer 3000) at a driving frequency of $1 \mathrm{~Hz}$.

\section{Results and discussion}

\section{1 Thermal analysis and phase evolution}

The typical thermal decomposition behavior of the BCZT raw material is shown in Fig. 1. The first weight loss at temperatures between 40 and $160{ }^{\circ} \mathrm{C}$ is due to desorption of physically adsorbed water (dehydration phenomena). The second weight loss in the range of 160-230 ${ }^{\circ} \mathrm{C}$ can be attributed to the chemisorbed

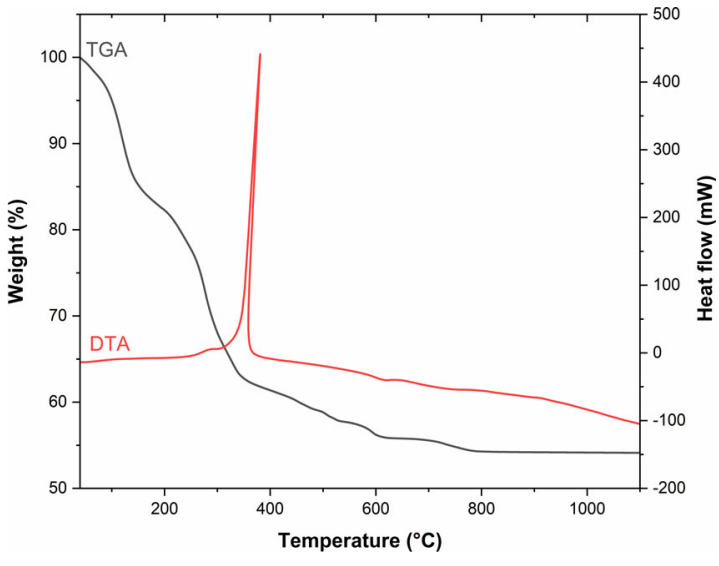

Fig. 1 TG-DTA curves of BCZT raw material.

water (dehydroxylation phenomena). The exothermic located peak at $380{ }^{\circ} \mathrm{C}$ is associated to the thermal decomposition of various organic compounds. The fourth loss between 580 and $800{ }^{\circ} \mathrm{C}$ is attributed to the formation and crystallization of BCZT phase [36]. Finally, the mass loss remains constant after $800{ }^{\circ} \mathrm{C}$. Based on these analyses, the temperature of $1000{ }^{\circ} \mathrm{C}$ was selected as the calcination temperature for the formation of highly pure perovskite BCZT.

\section{2 Morphology and phase characterization of rod- like BCZT powder}

In Fig. 2(a), a FE-SEM micrograph of the elaborated BCZT powder is presented. Besides very small amount of BCZT nanoparticles that were not yet transformed into BCZT rods, mainly rod-like grains with an average diameter and length of 0.45 and $4.25 \mu \mathrm{m}$, respectively, are visible with the corresponding aspect ratio around 10 .

The XRD pattern obtained for BCZT calcined powder is shown in Fig. 2(b). It was observed that BCZT powder was formed in a pure perovskite phase, without any trace of crystalline impurities. All diffraction peaks can be indexed based on the standard model X-ray polycrystalline tetragonal $\mathrm{BaTiO}_{3}$ (JCPDS card No. 96-901-4669) with the space group $P 4 \mathrm{~mm}$. The characteristic tetragonal reflection (110) was observed at $2 \theta=31.65^{\circ}$. BCZT powder has a tetragonal symmetry demonstrated by the peaks (002) and (200) splitting [37-40]. Insets of Fig. 2 clearly illustrate the splitting of these peaks in the $2 \theta$ range of $44^{\circ}-46^{\circ}$. Figure 2(c) depicts SAED patterns of BCZT calcined powder. The $d$-spacings calculated from the measured ring diameters (i.e., 2.834, 2.313, 1.999, and 1.634 $\AA$ ), correspond to (110), (111), (002), and (211) planes, respectively. These results are in good agreement with those obtained by XRD. 


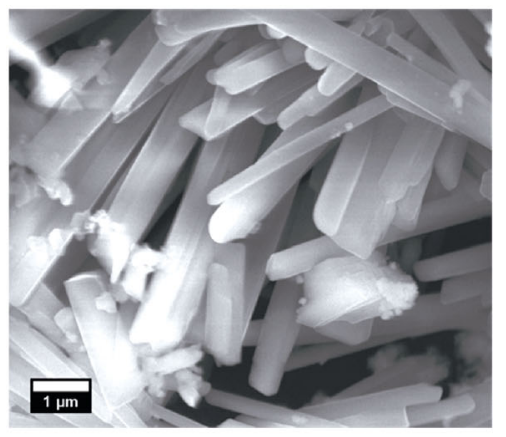

(a)

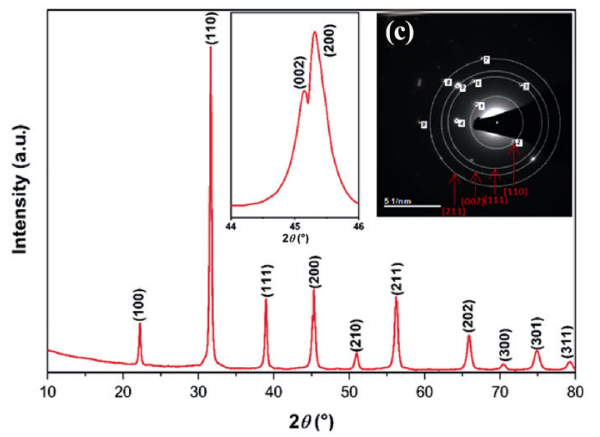

(b)

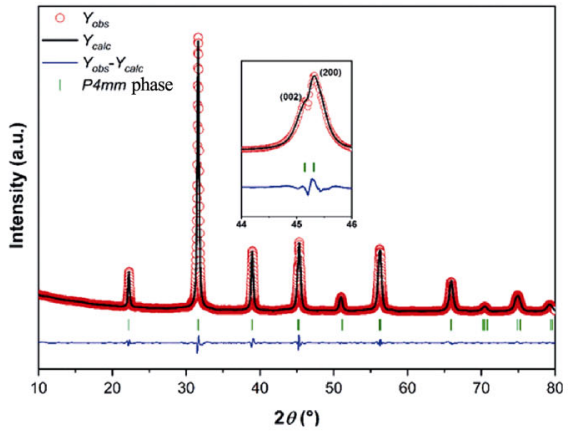

(d)

Fig. 2 (a) FE-SEM micrograph, (b) XRD, (c) SAED patterns, and (d) Rietveld refinement of BCZT powder calcined at $1000{ }^{\circ} \mathrm{C} / 4 \mathrm{~h}$.

Phase analysis of BCZT calcined powder was performed in the light of $P 4 \mathrm{~mm}$ space group as shown in Fig. 2(d). The refinement performed by FullProf Suite software indicates that the diffraction data of BCZT powder collected at room temperature mainly has a tetragonal phase $(P 4 m m) . Y_{\text {obs }}$ and $Y_{\text {calc }}$ correspond to the observed and calculated data respectively. In the Rietveld refinements, $\mathrm{Ba}$ and $\mathrm{Ca}$ ions are supposed to share the same crystallographic A-site of the BCZT perovskite structure. Similarly, $\mathrm{Ti}$ and $\mathrm{Zr}$ ions are positioned at the same B-site [40]. Table 1 indicates the lattice parameters, atomic positions, and space group obtained after refinement. These parameters are comparable to those reported in Refs. [40,41].

The degree of tetragonality $(c / a)$ at room temperature of BCZT is 1.0050. This value is in a good agreement with those of Liu and Ren [27] and Praveen et al. [40] which are 1.0050 and 1.0049 , respectively.

\section{3 Density measurements of rod-like BCZT ceramic}

Figure 3 displays SEM micrograph and the corresponding statistical distribution of length and diameter of BCZT ceramics sintered at $1250{ }^{\circ} \mathrm{C} / 10 \mathrm{~h}$. The microstructure reveals tightly bonded and well-defined rod-like grains with no obvious porosity, corresponding to a high bulk density of $5.50 \mathrm{~g} / \mathrm{cm}^{3}$ ( $95 \%$ of the theoretical density). The average length and diameter of the rod-like grains are 5.2 and $1.35 \mu \mathrm{m}$, respectively. Hence, an average aspect ratio is of 4 .

Table 1 Structural parameters obtained from Rietveld refinement of BCZT calcined powder

\begin{tabular}{|c|c|c|c|c|c|c|c|c|c|}
\hline Lattice parameter $(\AA)$ & Angle $\left(^{\circ}\right)$ & Volume $\left(\AA^{3}\right)$ & \multicolumn{4}{|c|}{ Atomic position $(x, y, z)$} & $\chi^{2}$ & Space group & $c / a$ \\
\hline$a=3.99420$ & \multirow{4}{*}{$\alpha=\beta=\gamma=90$} & \multirow{4}{*}{64.039} & $\mathrm{Ba} / \mathrm{Ca}$ & 0.00000 & 0.00000 & 0.01674 & \multirow{4}{*}{1.72} & \multirow{4}{*}{$P 4 m m$} & \multirow{4}{*}{1.0050} \\
\hline$b=3.99420$ & & & $\mathrm{Zr} / \mathrm{Ti}$ & 0.50000 & 0.50000 & 0.49641 & & & \\
\hline \multirow[t]{2}{*}{$c=4.01410$} & & & $\mathrm{O}_{1}$ & 0.50000 & 0.50000 & -0.03807 & & & \\
\hline & & & $\mathrm{O}_{2}$ & 0.50000 & 0.00000 & 0.55951 & & & \\
\hline
\end{tabular}
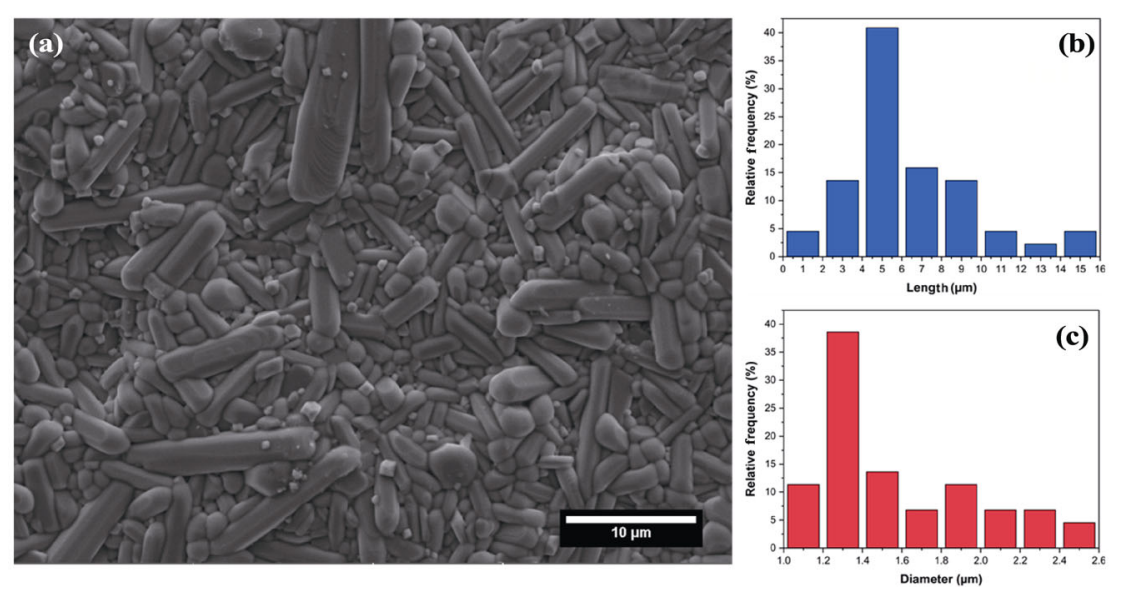

Fig. 3 (a) SEM micrograph and corresponding statistical distribution of (b) length and (c) diameter of BCZT ceramics sintered at $1250{ }^{\circ} \mathrm{C} / 10 \mathrm{~h}$. 


\section{4 Dielectric and ferroelectric properties of rod-like BCZT ceramics}

Figure 4(a) displays the temperature-dependence of the dielectric constant $\left(\varepsilon_{\mathrm{r}}\right)$ and the dielectric $\operatorname{loss}(\tan \delta)$ of rod-like BCZT ceramics at various frequencies from room temperature to $473 \mathrm{~K}$. The dielectric constant increases with increasing temperature and reaches the maximum at Curie temperature $\left(T_{\mathrm{c}}\right)$. A broad anomaly at $366 \mathrm{~K}$ associated to the tetragonal-cubic $(T-C)$ phase transition was observed $[31,42,43]$. The broad maximum observed is due to the overlap of several ferroelectric and non-ferroelectric regions [44]. Furthermore, with increasing frequency, the maximum dielectric constant decreases and Curie temperature shifts toward higher temperatures, indicating a typical relaxor frequency dispersion $[45,46]$.

The dielectric constant of normal ferroelectrics above $T_{\mathrm{c}}$ follows the Curie-Weiss law, as described by Eq. (1). The reciprocal dielectric constant was plotted as a function of temperature $(T)$ at $1 \mathrm{kHz}$ and fitted using Curie-Weiss law to evaluate this phase transition:

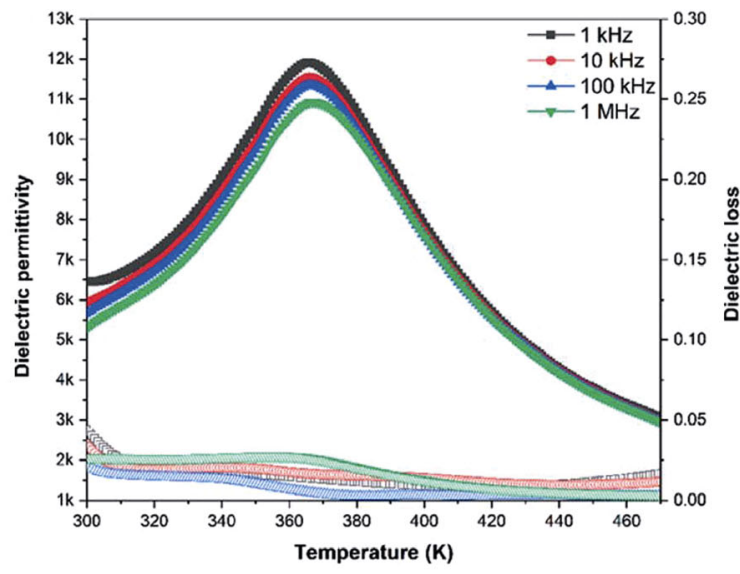

(a)

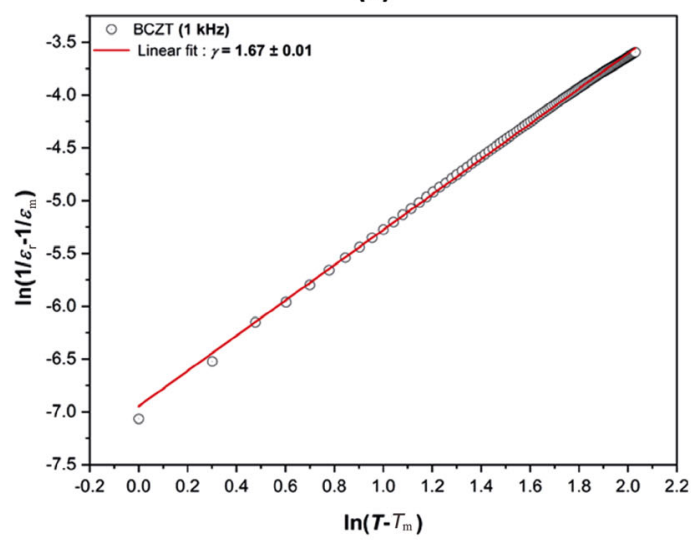

(c)

$$
\frac{1}{\varepsilon_{\mathrm{r}}}=\frac{T-T_{0}}{C} \quad\left(T>T_{0}\right)
$$

where $\varepsilon_{\mathrm{r}}$ is the real part of dielectric constant, $T_{0}$ is the Curie-Weiss temperature, and $C$ is the Curie-Weiss constant.

The plot of the inverse dielectric constant vs. temperature at $1 \mathrm{kHz}$ is shown in Fig. 4(b). The fitting results obtained by Eq. (1) are listed in Table 2. It was found that the real part of $\varepsilon_{\mathrm{r}}$ of BCZT ceramics deviates from the Curie-Weiss law above the Curie temperature. The deviations $\Delta T_{\mathrm{m}}$ as defined by Eq. (2):

$$
\Delta T_{\mathrm{m}}=T_{\mathrm{cw}}-T_{\mathrm{m}}
$$

where $T_{\mathrm{cw}}$ refers to the temperature from which $\varepsilon_{\mathrm{r}}$ starts to follow the Curie-Weiss law, and $T_{\mathrm{m}}$ denotes the temperature at which $\varepsilon_{\mathrm{r}}$ value reaches the maximum. The estimated value of deviations $\Delta T_{\mathrm{m}}$ is $47 \mathrm{~K}$.

To further confirm the degree of diffuseness in the BCZT ceramics, a modified empirical expression proposed by Uchino and Nomura [47] is used, which is given in Eq. (3):

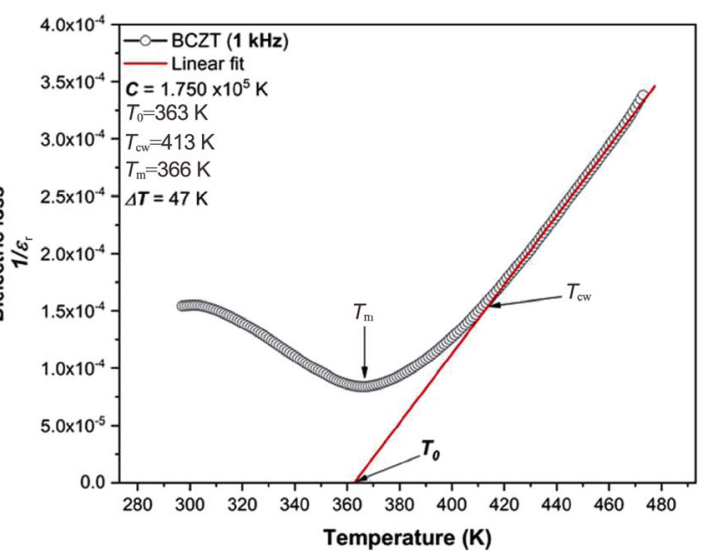

(b)

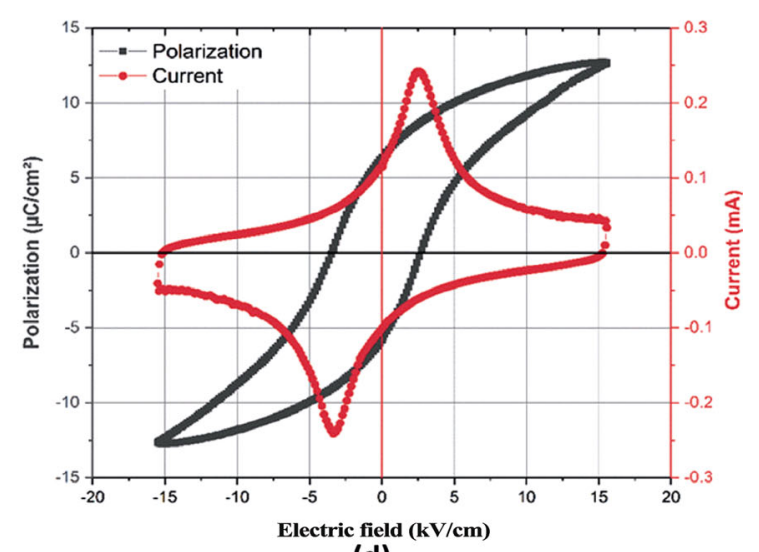

(d)

Fig. 4 (a) Temperature-dependence of dielectric constant and dielectric loss, (b, c) plots of Curie-Weiss relation and modified Curie-Weiss law to determine slope ( $\gamma$ ), and (d) $P-E$ and $I-E$ plots of BCZT ceramics. 


$$
\frac{1}{\varepsilon_{\mathrm{r}}}-\frac{1}{\varepsilon_{\mathrm{m}}}=\frac{\left(T-T_{0}\right)^{\gamma}}{C} \quad(1<\gamma<2)
$$

where $\varepsilon_{\mathrm{r}}$ and $\varepsilon_{\mathrm{m}}$ are the real part of the dielectric constant and its maximum value, respectively, and $\gamma$ (degree of diffuse transitions) and $C$ are constants. For an ideal relaxor ferroelectric $\gamma=2$, while for a normal ferroelectric $\gamma=1$ and the system follows the CurieWeiss law [48].

Figure 4(c) depicts the linear fitting plot of $\ln \left(1 / \varepsilon_{\mathrm{r}}-\right.$ $\left.1 / \varepsilon_{\mathrm{m}}\right)$ vs. $\ln \left(T-T_{\mathrm{m}}\right)$ at $1 \mathrm{kHz}$ for BCZT ceramics. After curve fitting by using Eq. (3), the value obtained for $\gamma$ at $1 \mathrm{kHz}$ in BCZT ceramics is 1.67 .

Previously, we reported the synthesis of BCZT ceramics using CTAB surfactant at different concentrations [31]. It was found that the small spheroidal grains $(60-1000 \mathrm{~nm})$ were distributed at the boundaries of coarse grains and occupied the pores created during the sintering to create high dense ceramics with a relative density $\left(D_{\mathrm{r}}\right)$ of about $95.2 \%$ equal to that of BCZT ceramics with rod-like grains. The dielectric constant and the dielectric loss of BCZT ceramics with spherical grains were found to be 7584 and 0.0158 , respectively (Table 2). However, the dielectric constant and the dielectric loss in BCZT ceramics with rod-like grains reached 11,906 and 0.014 , respectively. Hence, tailoring BCZT ceramics with large aspect ratio could enhance its dielectric properties [49,50].

$P-E$ and $I-E$ plots of BCZT ceramics at room temperature are represented in Fig. 4(d). It displays a slim $P-E$ hysteresis loop with remnant polarization $\left(P_{\mathrm{r}}\right)$ of $6.01 \mu \mathrm{C} / \mathrm{cm}^{2}$ and coercive field $\left(E_{\mathrm{c}}\right)$ of $2.46 \mathrm{kV} / \mathrm{cm}$. The $I-E$ curve shows two distinct peaks around the coercive fields, indicating the domain switching phenomena [51]. The appearance of a domain switching current peak (Fig. 4(d)), with an application of electric field confirms the ferroelectric nature of BCZT ceramics. The $E_{\mathrm{c}}$ can be evaluated from the polarization vs. electric field $(P-E)$ hysteresis loop or by the current vs. electric field $(I-E)$ curve [52].

\section{5 Electrocaloric effect in rod-like BCZT ceramics}

To evaluate the effect of grain shape tailoring on the electrocaloric effect (ECE) in the rod-like BCZT ceramics, Fig. 5(a) depicts $P-E$ hysteresis loops registered at different temperatures at the driving frequency of $1 \mathrm{~Hz}$, as in our recent paper Ref. [25]. The same frequency (1 $\mathrm{Hz}$ ) was kept to avoid any influence of the frequency as reported by Cheng et al. [53]. With increasing the temperature, the remnant polarization decreases continuously due to the disappearance of ferroelectric domains [54].

After recording $P-E$ hysteresis loops as a function of the temperature, we perform a seven-order polynomial fitting by considering only the upper branches of these curves (Fig. 5(a)). Then, the variation of the polarization vs. the temperature at every fixed applied electric field can be deduced (Fig. 5(b)). It was noticed that the polarization decreases slowly from room temperature, then drops rapidly with the temperature around $T_{\mathrm{c}}$.

Table 2 Dielectric properties of BCZT ceramics with rod-like and spherical-like grains

\begin{tabular}{ccccccccccccc}
\hline & $\varepsilon_{\max }$ & $\tan \delta$ & $D_{\mathrm{r}}(\%)$ & $T_{0}(\mathrm{~K})$ & $C\left(10^{5} \mathrm{~K}\right)$ & $T_{\mathrm{m}}(\mathrm{K})$ & $T_{\mathrm{cw}}(\mathrm{K})$ & $\Delta T_{\mathrm{m}}(\mathrm{K})$ & $\gamma$ & Ref. \\
\hline BCZT rod-like & 11,906 & 0.014 & 95 & 363 & 1.75 & 366 & 413 & 47 & 1.67 & This study \\
BCZT sphere-like & 7584 & 0.0158 & 95.2 & 373 & 1.812 & 364 & 418 & 54 & 1.79 & {$[31]$} \\
\hline
\end{tabular}

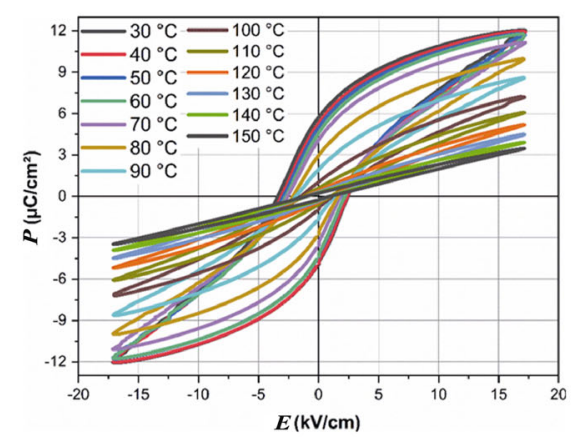

(a)

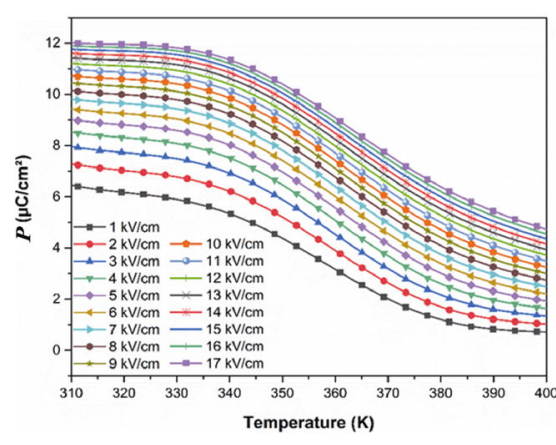

(b)

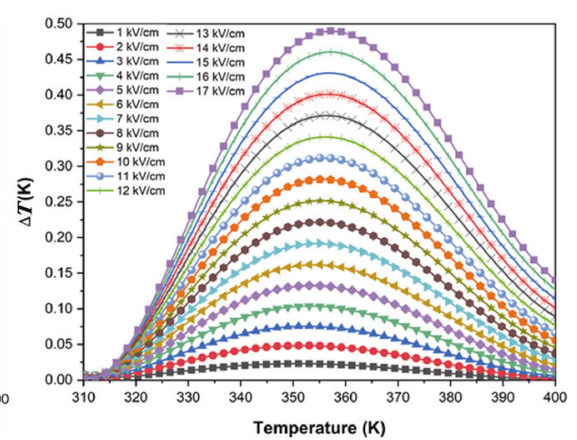

(c)

Fig. 5 Temperature-dependence of (a) $P-E$ loops, (b) electric polarization, and (c) the reversible adiabatic electrocaloric temperature change $(\Delta T)$ of rod-like BCZT ceramics under various applied electric fields. 
$\Delta T$ was calculated by indirect method based on Maxwell equation that leads to Eq. (4).

$$
\Delta T=-\int_{E_{1}}^{E_{2}} \frac{T}{\rho C_{\mathrm{p}}}\left(\frac{\partial P}{\partial T}\right)_{E} \mathrm{~d} E
$$

where $\rho$ and $C_{\mathrm{p}}$ are the mass density and the specific heat of the material, respectively. The value of $C_{\mathrm{p}}$ $(0.378 \mathrm{~J} /(\mathrm{g} \cdot \mathrm{K}))$ was taken from Ref. [26].

Figure 5(c) shows the temperature-dependence of $\Delta T$. Each curve corresponds to a fixed applied electric field and evidences the ferroelectric-paraelectric phase transition with temperature increasing. The highest value obtained for $\Delta T$ was $0.492 \mathrm{~K}$ at $17 \mathrm{kV} / \mathrm{cm}$ at the tetragonal-cubic transition around $360 \mathrm{~K}$. It is worth noting that a further increase in the applied external electric field could enhance the $\Delta T$ value of the material since the $P-E$ loops have not reached saturation yet. Commonly, the electrocaloric responsivity $(\zeta=$ $\Delta T / \Delta E$ ) is used for more suitable evaluation of the electrocaloric effect of materials. The obtained $\Delta T$ value at $17 \mathrm{kV} / \mathrm{cm}$ corresponds to the electrocaloric responsivity of $\zeta=0.289(\mathrm{~K} \cdot \mathrm{mm}) / \mathrm{kV}$.

Our findings are compared with previously published results on BCZT ceramics in Table 3 in which the electrocaloric properties ( $\Delta T$ and $\zeta$ ) of BCZT lead-free ceramics with different compositions and shapes are listed. It should be pointed out that $\Delta T$ value in the studied rod-like BCZT ceramics was found larger than other reported values in literature [22,37]. However, its electrocaloric responsivity is among the average values. This can be attributed to the elaboration method, the chemical composition, atomic rate, and site occupancy in the structure. It is well known that the nature of the precursors used for the syntheses often plays a key role

Table 3 Comparison of the electrocaloric properties of rod-like BCZT ceramics with other lead-free BCZT ceramics reported in literature

\begin{tabular}{cccccc}
\hline Ceramic & $\Delta T(\mathrm{~K}) \Delta E(\mathrm{kV} / \mathrm{cm})$ & $T(\mathrm{~K})$ & $\zeta((\mathrm{K} \cdot \mathrm{mm}) / \mathrm{kV})$ & Ref. \\
\hline $\mathrm{Ba}_{0.85} \mathrm{Ca}_{0.15} \mathrm{Zr}_{0.10} \mathrm{Ti}_{0.90} \mathrm{O}_{3}$ & 0.492 & 17 & 360 & 0.289 & $\begin{array}{c}\text { This } \\
\text { study }\end{array}$ \\
$\mathrm{Ba}_{0.85} \mathrm{Ca}_{0.15} \mathrm{Zr}_{0.10} \mathrm{Ti}_{0.90} \mathrm{O}_{3}$ & 0.118 & 6.65 & 363 & 0.164 & {$[25]$} \\
$\mathrm{Ba}_{0.85} \mathrm{Ca}_{0.15} \mathrm{Zr}_{0.10} \mathrm{Ti}_{0.90} \mathrm{O}_{3}$ & 0.4 & 21.5 & 370 & 0.186 & {$[37]$} \\
$\mathrm{Ba}_{0.85} \mathrm{Ca}_{0.15} \mathrm{Zr}_{0.10} \mathrm{Ti}_{0.90} \mathrm{O}_{3}$ & 0.152 & 8 & 373 & 0.19 & {$[26]$} \\
$\mathrm{Ba}_{0.80} \mathrm{Ca}_{0.2} \mathrm{Zr}_{0.04} \mathrm{Ti}_{0.96} \mathrm{O}_{3}$ & 0.27 & 7.95 & 386 & 0.340 & {$[22]$} \\
$\mathrm{Ba}_{0.80} \mathrm{Ca}_{0.20} \mathrm{Zr}_{0.08} \mathrm{Ti}_{0.92} \mathrm{O}_{3}$ & 0.22 & 7.95 & 386 & 0.27 & {$[61]$} \\
$\mathrm{Ba}_{0.91} \mathrm{Ca}_{0.09} \mathrm{Zr}_{0.14} \mathrm{Ti}_{0.86} \mathrm{O}_{3}$ & 0.3 & 20 & 328 & 0.150 & {$[62]$} \\
$\mathrm{Ba}_{0.92} \mathrm{Ca}_{0.08} \mathrm{Zr}_{0.05} \mathrm{Ti}_{0.95} \mathrm{O}_{3}$ & 0.38 & 15 & 410 & 0.253 & {$[61]$} \\
\hline
\end{tabular}

in determining the shape and size of the nanomaterials. The nucleation and growth of BCZT from the molecular, ionic, or oxide titanium/zirconium precursors in the presence of barium/calcium carbonates or salts are often dictated by the nature of the cubic crystal structure, which results in the formation of BCZT nanocrystals with spherical or cubic shapes with aspect ratio of about 1 [55]. However, in this study, the CTAB surfactant was used as directing agent to produce BCZT ceramics with rod-like grains.

The ECE is associated with the polarization, phenomenological coefficient, multiple polar states, as well as the breakdown electric field, etc. [56-59]. Indeed, these physical properties are strongly dependent on features and morphologies of dielectric materials such as grain size, size distribution, and aspect ratio. As reported by Zhang et al. [60], the electrocaloric properties in $\mathrm{Ba}_{0.67} \mathrm{Sr}_{0.33} \mathrm{TiO}_{3} /$ Poly (vinylidene fluorideertrifluoroethylene-ter-chlorofluoroethylene) (BST/P (VDFTrFE-CFE)) nanocomposites by embedding BST fillers with nanoparticles, cubes, rods, and nanowires in morphology are closely related to the aspect ratio of the ceramic BST nanofillers [60]. They found that fillers with high aspect ratio exhibit much higher breakdown and greater ECE. Likewise, Tang et al. [49] demonstrated that using high-aspect-ratio of $\mathrm{BaTiO}_{3}$ (BT) nanowires is an effective way to control and improve the dielectric performance of (BT/PVDF) nanocomposites. Analogically, the enhanced electrocaloric effect in our sample could be attributed to high aspect ratio (4) of BCZT rod-like ceramics as compared with other reported BCZT samples exhibiting almost spherical grains (aspect ratio $\approx 1$ ). Thus, BCZT ceramics with rod-like grains have good potential to be used for the application in eco-friendly solid-state cooling devices.

\section{Conclusions}

In this study, lead-free rod-like BCZT ceramics have been successfully prepared by CTAB-assisted solvothermal processing. XRD and SAED revealed that rod-like BCZT ceramics exhibit pure perovskite phase with tetragonal symmetry and a remarkable $c / a$ of 1.0050 and a high density (95\%). SEM micrograph depicted that BCZT ceramics are self-assembled into rod-like grain with an average length and diameter of 5.2 and $1.35 \mu \mathrm{m}$, respectively, and therefore with an aspect ratio of 4 . Rod-like BCZT ceramics demonstrated 
enhanced dielectric $\left(\varepsilon_{\mathrm{r}}=11,906, \tan \delta=0.014\right)$ and ferroelectric $\left(P_{\mathrm{r}}=6.01 \mu \mathrm{C} / \mathrm{cm}^{2}\right.$ and $\left.E_{\mathrm{c}}=2.46 \mathrm{kV} / \mathrm{cm}\right)$ properties compared to BCZT ceramics with spherical grains. Likewise, around $360 \mathrm{~K}, \Delta T$ and responsivity $\zeta$ were found to be $0.492 \mathrm{~K}$ and $0.289(\mathrm{~K} \cdot \mathrm{mm}) / \mathrm{kV}$, respectively, which are larger than those found in the reported BCZT ceramics. Therefore, lead-free BCZT ceramics with high aspect ratio could be a potential candidate for cooling material in novel environmentally friendly solid-state cooling devices.

\section{Acknowledgements}

The authors gratefully acknowledge the generous financial support of CNRST Priority Program (PPR 15/2015), Slovenian Research Agency Program (P1-0125), and European Union's Horizon 2020 Research and Innovation Program under the Marie Skłodowska-Curie Grant Agreement (No. 778072).

\section{References}

[1] Kitanovski A, Plaznik U, Tome U, et al. Present and future caloric refrigeration and heat-pump technologies. Int $J$ Refrig 2015, 57: 288-298.

[2] Suchaneck G, Pakhomov O, Gerlach G. Electrocaloric cooling. In Refrigeration. Orhan E, Ed. InTechOpen, 2017.

[3] Mathur ND. Future trends in electrocalorics materials. In Electrocaloric Materials. Correia T, Zhang Q, Eds. Berlin, Heidelberg: Springer Berlin Heidelberg, 2013: 251-253.

[4] Zhang J, Xuan YM. An integrated design of the photovoltaic-thermoelectric hybrid system. Sol Energy 2019, 177: 293-298.

[5] Lu X, Zhao DL, Ma T, et al. Thermal resistance matching for thermoelectric cooling systems. Energy Convers Manag 2018, 169: 186-193.

[6] Taboada-Moreno CA, Sánchez-De Jesús F, Pedro-García F, et al. Large magnetocaloric effect near to room temperature in $\mathrm{Sr}$ doped $\mathrm{La}_{0.7} \mathrm{Ca}_{0.3} \mathrm{MnO}_{3}$. J Magn Magn Mater 2020, 496: 165887.

[7] Du HL, Chang YF, Li CW, et al. Ultrahigh room temperature electrocaloric response in lead-free bulk ceramicsviatape casting. J Mater Chem C 2019, 7: 6860-6866.

[8] Ožbolt M, Kitanovski A, Tušek J, et al. Electrocaloric vs. magnetocaloric energy conversion. Int J Refrig 2014, 37: 16-27.

[9] Quintero M, Ghivelder L, Gomez-Marlasca F, et al. Decoupling electrocaloric effect from Joule heating in a solid state cooling device. Appl Phys Lett 2011, 99: 232908.

[10] Kutnjak Z, Rožič B, Pirc R. Wiley Encyclopedia of Electrical and Electronics Engineering. In Electrocaloric Effect: Theory, Measurements, and Applications. Hoboken,
NJ, USA: John Wiley \& Sons, Inc., 2015: 1-19.

[11] Tuttle BA, Payne DA. The effects of microstructure on the electrocaloric properties of $\mathrm{Pb}(\mathrm{Zr}, \mathrm{Sn}, \mathrm{Ti}) \mathrm{O}_{3}$ ceramics. Ferroelectrics 1981, 37: 603-606.

[12] Qiu JH, Jiang Q. Effect of electric field on electrocaloric effect in $\mathrm{Pb}\left(\mathrm{Zr}_{1-x} \mathrm{Ti}_{x}\right) \mathrm{O}_{3}$ solid solution. Phys Lett A 2008, 372: 7191-7195.

[13] Xiao D, Wang Y, Zhang R, et al. Electrocaloric properties of $(1-x) \mathrm{Pb}\left(\mathrm{Mg}_{1 / 3} \mathrm{Nb}_{2 / 3}\right) \mathrm{O}_{3-x} \mathrm{PbTiO}_{3}$ ferroelectric ceramics near room temperature. Mater Chem Phys 1998, 57: 182-185.

[14] Gao JH, Xue DZ, Liu WF, et al. Recent progress on $\mathrm{BaTiO}_{3}$-based piezoelectric ceramics for actuator applications. Actuators 2017, 6: 24.

[15] Panda PK, Sahoo B. PZT to lead free piezo ceramics: A review. Ferroelectrics 2015, 474: 128-143.

[16] Rödel J, Jo W, Seifert KTP, et al. Perspective on the development of lead-free piezoceramics. J Am Ceram Soc 2009, 92: 1153-1177.

[17] Puli VS, Pradhan DK, Chrisey DB, et al. Structure, dielectric, ferroelectric, and energy density properties of $(1-x) \mathrm{BZT}-x \mathrm{BCT}$ ceramic capacitors for energy storage applications. J Mater Sci 2013, 48: 2151-2157.

[18] Hu PH, Shen Y, Guan YH, et al. Topological-structure modulated polymer nanocomposites exhibiting highly enhanced dielectric strength and energy density. Adv Funct Mater 2014, 24: 3172-3178.

[19] Coondoo I, Panwar N, Amorín H, et al. Synthesis and characterization of lead-free $0.5 \mathrm{Ba}\left(\mathrm{Zr}_{0.2} \mathrm{Ti}_{0.8}\right) \mathrm{O}_{3}-0.5\left(\mathrm{Ba}_{0.7}\right.$ $\left.\mathrm{Ca}_{0.3}\right) \mathrm{TiO}_{3}$ ceramic. J Appl Phys 2013, 113: 214107.

[20] Wang FJ, Li W, Jiang HL, et al. Preparation and dielectric properties of $\mathrm{Ba}_{0.95} \mathrm{Ca}_{0.05} \mathrm{Ti}_{0.8} \mathrm{Zr}_{0.2} \mathrm{O}_{3}$-polyethersulfone composites. J Appl Phys 2010, 107: 043528.

[21] Liu X, Wu D, Chen Z, et al. Ferroelectric, dielectric and pyroelectric properties of $\mathrm{Sr}$ and $\mathrm{Sn}$ codoped BCZT lead free ceramics. Adv Appl Ceram 2015, 114: 436-441.

[22] Asbani B, Dellis JL, Lahmar A, et al. Lead-free $\mathrm{Ba}_{0.8} \mathrm{Ca}_{0.2}\left(\mathrm{ZrxTi}_{1-x}\right) \mathrm{O}_{3}$ ceramics with large electrocaloric effect. Appl Phys Lett 2015, 106: 042902.

[23] Luo BC, Wang XH, Wang YP, et al. Fabrication, characterization, properties and theoretical analysis of ceramic/PVDF composite flexible films with high dielectric constant and low dielectric loss. J Mater Chem A 2014, 2: 510-519.

[24] Hanani Z, Ablouh EH, Amjoud M', et al. Very-low temperature synthesis of pure and crystalline lead-free $\mathrm{B}_{0.85} \mathrm{C}_{0.15} \mathrm{Zr}_{0.1} \mathrm{Ti}_{0.9} \mathrm{O}_{3}$ ceramic. Ceram Int 2018, 44: $10997-$ 11000 .

[25] Hanani Z, Mezzane D, Amjoud M, et al. Phase transitions, energy storage performances and electrocaloric effect of the lead-free $\mathrm{Ba}_{0.85} \mathrm{Ca}_{0.15} \mathrm{Zr}_{0.10} \mathrm{Ti}_{0.90} \mathrm{O}_{3}$ ceramic relaxor. $J$ Mater Sci: Mater Electron 2019, 30: 6430-6438.

[26] Kaddoussi H, Lahmar A, Gagou Y, et al. Sequence of structural transitions and electrocaloric properties in $\left(\mathrm{Ba}_{1-x} \mathrm{Ca}_{x}\right)\left(\mathrm{Zr}_{0.1} \mathrm{Ti}_{0.9}\right) \mathrm{O}_{3}$ ceramics. J Alloys Compd 2017, 
713: $164-179$

[27] Liu WF, Ren XB. Large piezoelectric effect in $\mathrm{Pb}$-free ceramics. Phys Rev Lett 2009, 103: 257602.

[28] Bai Y, Matousek A, Tofel P, et al. $(\mathrm{Ba}, \mathrm{Ca})(\mathrm{Zr}, \mathrm{Ti}) \mathrm{O}_{3}$ lead-free piezoelectric ceramics-The critical role of processing on properties. J Eur Ceram Soc 2015, 35: 3445-3456.

[29] Hao JG, Bai WF, Li W, et al. Correlation between the microstructure and electrical properties in high-performance $\left(\mathrm{Ba}_{0.85} \mathrm{Ca}_{0.15}\right)\left(\mathrm{Zr}_{0.1} \mathrm{Ti}_{0.9}\right) \mathrm{O}_{3}$ lead-free piezoelectric ceramics. J Am Ceram Soc 2012, 95: 1998-2006.

[30] Shi J, Lu XY, Shao JH, et al. Effects on structure and properties of BCZT lead-free piezoelectric ceramics by rare-earth doping. Ferroelectrics 2017, 507: 186-197.

[31] Hanani Z, Mezzane D, Amjoud M, et al. Enhancement of dielectric properties of lead-free BCZT ferroelectric ceramics by grain size engineering. Superlattices Microstruct 2019, 127: 109-117.

[32] Zhao PJ, Wang L, Bian L, et al. Growth mechanism, modified morphology and optical properties of coral-like $\mathrm{BaTiO}_{3}$ architecture through CTAB assisted synthesis. $J$ Mater Sci Technol 2015, 31: 223-228.

[33] Wang YX, Sun J, Fan XY, et al. A CTAB-assisted hydrothermal and solvothermal synthesis of $\mathrm{ZnO}$ nanopowders. Ceram Int 2011, 37: 3431-3436.

[34] Yayapao O, Thongtem $\mathrm{T}$, Phuruangrat $\mathrm{A}$, et al. CTAB-assisted hydrothermal synthesis of tungsten oxide microflowers. J Alloys Compd 2011, 509: 2294-2299.

[35] Vijayalakshmi R, Rajendran V. Impact of surfactants on physical properties of $\mathrm{BaTiO}_{3}$ nanoparticles. Int $J$ Nanosci 2013, 12: 1350003 .

[36] Praveen JP, Karthik T, James AR, et al. Effect of poling process on piezoelectric properties of sol-gel derived BZT-BCT ceramics. J Eur Ceram Soc 2015, 35: 1785-1798.

[37] Patel S, Sharma P, Vaish R. Enhanced electrocaloric effect in $\mathrm{Ba}_{0.85} \mathrm{Ca}_{0.15} \mathrm{Zr}_{0.1} \mathrm{Ti}_{0.9-x} \mathrm{Sn}_{x} \mathrm{O}_{3}$ ferroelectric ceramics. Phase Transit 2016, 89: 1062-1073.

[38] Roy S, Maharana R, Reddy SR, et al. Structural, ferroelectric and piezoelectric properties of chemically processed, low temperature sintered piezoelectric BZT-BCT ceramics. Mater Res Express 2016, 3: 035702.

[39] Xu HR, Gao L, Guo JK. Preparation and characterizations of tetragonal barium titanate powders by hydrothermal method. J Eur Ceram Soc 2002, 22: 1163-1170.

[40] Praveen JP, Karthik T, James AR, et al. Effect of poling process on piezoelectric properties of sol-gel derived BZT-BCT ceramics. J Eur Ceram Soc 2015, 35: 1785-1798.

[41] Jeong IK, Ahn JS. The atomic structure of lead-free $\mathrm{Ba}\left(\mathrm{Zr}_{0.2} \mathrm{Ti}_{0.8}\right) \mathrm{O}_{3}-\left(\mathrm{Ba}_{0.7} \mathrm{Ca}_{0.3}\right) \mathrm{TiO}_{3}$ by using neutron total scattering analysis. Appl Phys Lett 2013, 102: 179903.

[42] Wang ZM, Wang JJ, Chao XL, et al. Synthesis, structure, dielectric, piezoelectric, and energy storage performance of $\left(\mathrm{Ba}_{0.85} \mathrm{Ca}_{0.15}\right)\left(\mathrm{Ti}_{0.9} \mathrm{Zr}_{0.1}\right) \mathrm{O}_{3}$ ceramics prepared by different methods. J Mater Sci: Mater Electron 2016, 27: 5047-5058.
[43] Bai WF, Chen DQ, Zhang JJ, et al. Phase transition behavior and enhanced electromechanical properties in $\left(\mathrm{Ba}_{0.85} \mathrm{Ca}_{0.15}\right)\left(\mathrm{Zr}_{x} \mathrm{Ti}_{1-x}\right) \mathrm{O}_{3}$ lead-free piezoceramics. Ceram Int 2016, 42: 3598-3608.

[44] Venkata RE, Mahajan A, Graça MPF, et al. Structure and ferroelectric studies of $\left(\mathrm{Ba}_{0.85} \mathrm{Ca}_{0.15}\right)\left(\mathrm{Ti}_{0.9} \mathrm{Zr}_{0.1}\right) \mathrm{O}_{3}$ piezoelectric ceramics. Mater Res Bull 2013, 48: 4395-4401.

[45] Bokov AA, Ye ZG. Recent progress in relaxor ferroelectrics with perovskite structure. In Frontiers of Ferroelectricity. Boston, MA: Springer US, 2007: 31-52.

[46] Liu YW, Pu YP, Sun ZX. Enhanced relaxor ferroelectric behavior of BCZT lead-free ceramics prepared by hydrothermal method. Mater Lett 2014, 137: 128-131.

[47] Uchino K, Nomura S. Critical exponents of the dielectric constants in diffused-phase-transition crystals. Ferroelectr Lett Sect 1982, 44: 55-61.

[48] Uchino K. Advanced Piezoelectric Materials. In Relaxor Ferroelectric-Based Ceramics. Elsevier, 2017: 127-153.

[49] Tang HX, Zhou Z, Sodano HA. Relationship between $\mathrm{BaTiO}_{3}$ nanowire aspect ratio and the dielectric permittivity of nanocomposites. ACS Appl Mater Interfaces 2014, 6: 5450-5455.

[50] Chary KS, Panda HS, Prasad CD. Fabrication of large aspect ratio $\mathrm{Ba}_{0.85} \mathrm{Ca}_{0.15} \mathrm{Zr}_{0.1} \mathrm{Ti}_{0.9} \mathrm{O}_{3}$ superfine fibers-based flexible nanogenerator device: Synergistic effect on curie temperature, harvested voltage, and power. Ind Eng Chem Res 2017, 56: 10335-10342.

[51] Kumar A, Bhanu Prasad VV, James Raju KC, et al. Optimization of poling parameters of mechanically processed PLZT 8/60/40 ceramics based on dielectric and piezoelectric studies. Eur Phys $J$ B 2015, 88: 287.

[52] Zhan D, Xu Q, Huang DP, et al. Dielectric nonlinearity and electric breakdown behaviors of $\mathrm{Ba}_{0.95} \mathrm{Ca}_{0.05} \mathrm{Zr}_{0.3} \mathrm{Ti}_{0.7} \mathrm{O}_{3}$ ceramics for energy storage utilizations. $J$ Alloys Compd 2016, 682: 594-600.

[53] Cheng XY, Weyland F, Novak N, et al. Indirect electrocaloric evaluation: Influence of polarization hysteresis measurement frequency. Phys Status Solidi A 2019, 216: 1900684

[54] Hamza A, Benabdallah F, Kallel I, et al. Effect of rare-earth substitution on the electrical properties and Raman spectroscopy of BCTZ ceramics. J Alloys Compd 2018, 735: 2523-2531.

[55] Chen XF, Chao XL, Yang ZP. Submicron Barium calcium zirconium titanate ceramic for energy storage synthesised via the co-precipitation method. Mater Res Bull 2019, 111: 259-266.

[56] Lu B, Yao YB, Jian XD, et al. Enhancement of the electrocaloric effect over a wide temperature range in PLZT ceramics by doping with $\mathrm{Gd}^{3+}$ and $\mathrm{Sn}^{4+}$ ions. J Eur Ceram Soc 2019, 39: 1093-1102.

[57] Jian XD, Lu B, Li DD, et al. Direct measurement of large electrocaloric effect in $\mathrm{Ba}\left(\mathrm{ZrxTi}_{1-x}\right) \mathrm{O}_{3}$ ceramics. ACS Appl Mater Interfaces 2018, 10: 4801-4807. 
[58] Lu B, Li PL, Tang ZH, et al. Large electrocaloric effect in relaxor ferroelectric and antiferroelectric lanthanum doped lead zirconate titanate ceramics. Sci Rep 2017, 7: 45335.

[59] Li XY, Lu SG, Chen XZ, et al. Pyroelectric and electrocaloric materials. J Mater Chem C 2013, 1: 23-37.

[60] Zhang GZ, Zhang XS, Yang TN, et al. Colossal roomtemperature electrocaloric effect in ferroelectric polymer nanocomposites using nanostructured Barium strontium titanates. ACS Nano 2015, 9: 7164-7174.

[61] Singh G, Tiwari VS, Gupta PK. Electro-caloric effect in $\left(\mathrm{Ba}_{1-x} \mathrm{Ca}_{x}\right)\left(\mathrm{Zr}_{0.05} \mathrm{Ti}_{0.95}\right) \mathrm{O}_{3}$ : A lead-free ferroelectric material. Appl Phys Lett 2013, 103: 202903.

[62] Bai Y, Han X, Qiao LJ. Optimized electrocaloric refrigeration capacity in lead-free $(1-x) \mathrm{BaZr}_{0.2} \mathrm{Ti}_{0.8} \mathrm{O}_{3}-x \mathrm{Ba}_{0.7} \mathrm{Ca}_{0.3} \mathrm{TiO}_{3}$ ceramics. Appl Phys Lett 2013, 102: 252904.
Open Access This article is licensed under a Creative Commons Attribution 4.0 International License, which permits use, sharing, adaptation, distribution and reproduction in any medium or format, as long as you give appropriate credit to the original author(s) and the source, provide a link to the Creative Commons licence, and indicate if changes were made.

The images or other third party material in this article are included in the article's Creative Commons licence, unless indicated otherwise in a credit line to the material. If material is not included in the article's Creative Commons licence and your intended use is not permitted by statutory regulation or exceeds the permitted use, you will need to obtain permission directly from the copyright holder.

To view a copy of this licence, visit http://creativecommons. org/licenses/by/4.0/. 